\title{
Exploiting Reversibility in the Complete Simulation of Reversible Circuits
}

\author{
Robert Wille*† Simon Stelter* $\quad$ Rolf Drechsler*† \\ *Institute of Computer Science, University of Bremen, 28359 Bremen, Germany \\ †Cyber-Physical Systems, DFKI GmbH, 28359 Bremen, Germany \\ \{rwille,sstelter,drechsle\}@informatik.uni-bremen.de
}

\begin{abstract}
Reversible circuits employ an alternative computation paradigm where all operations are performed in a reversible fashion only. Motivated by the promising applications, researchers started to developed corresponding design methods for this kind of circuits. In most of the resulting solutions, they try to address the restrictions and limitations that come with this alternative computation paradigm. In this work, we are instead showing possible advantages to be exploited. We present an alternative solution for complete simulation of reversible circuits which explicitly utilizes the reversibility of the underlying computation paradigm. By this, improvements of up to three orders of magnitude compared to the standard simulation can be achieved.
\end{abstract}

\section{INTRODUCTION}

From the beginning, researchers and engineers narrowed the investigation of computing machines down to a preponderantly irreversible computing paradigm. In fact, most of the established computations are not invertible. A simple standard operation like the logical AND already illustrates that. Indeed, it is possible to obtain the inputs of an AND gate if the output is set to 1 (then, both inputs must be set to 1 as well). But, it is not possible to determine the input values if the AND outputs 0 . While mainly relying on this conventional way of computation, alternative paradigms and their applications have hardly been considered and exploited yet.

A promising alternative is based on reversible computation, a computing paradigm which allows bijective operations only, i.e. reversible $n$-input $n$-output functions that map each possible input vector to a unique output vector. In circuits based on reversible logic, all computations can be reverted (i.e. the inputs can be obtained from the outputs and vice versa). For this purpose, established conventional gate libraries can obviously not be applied. As a consequence, new libraries of reversible gates have been introduced. Albeit not so well established yet, reversible computation enables several promising applications and, indeed, superiors conventional computation paradigms in many domains including but not limited to:

- Low Power Computation, where the fact that no information is lost in reversible computation can be exploited (see e.g. [1], [2], [3]),

- Adiabatic Circuits, a special low power technology where reversible circuits are particularly suited for (see e.g. [4]),
- Encoding and Decoding Devices, which always realize one-to-one mappings and, thus, inherently follow a reversible computing paradigm (see e.g. [5]),

- Quantum Computation, which enables to solve many relevant problems significantly faster than conventional circuits and inherently is reversible (see e.g. [6]), and

- Program Inversion (see e.g. [7]), as programs based on a reversible computation paradigm would allow an inherent and obvious program inversion.

Motivated by these applications, researchers started the investigation of corresponding methods for computer-aided design of this kind of circuits. This led to a variety of design solutions for a wide range of design tasks such as synthesis (see e.g. [8], [9], [10], [11]), optimization (see e.g. [12], [13]), verification (see e.g. [14], [15]), debugging (see e.g. [16]), and even automatic test pattern generation (see e.g. [17], [18]). Good overviews can be found e.g. in [19], [20], [21].

In most of these approaches, researchers try to address the restrictions and differences caused by reversible circuits in comparison to their conventional counterparts. For example, fanout and feedback are not directly allowed and, hence, the respective circuits have to be composed as cascades of special reversible gates. But beyond that, the reversible computing paradigm also employs several advantages that can be exploited in the design.

In this paper, we make use of one of these advantages in order to improve the complete simulation of reversible circuits. In fact, we present a revised simulation approach that does not consider each pattern to be simulated separately, but all possible patterns at once. We show that such a consideration is beneficial since, in reversible computation, (1) usually just a very small set of patterns is affected by a gate and (2) gates simply lead to an exchange of input patterns. The proposed solution significantly reduces the complexity of simulation and, hence, allows for speed-ups of up to three orders of magnitude.

In the remainder of this paper, the proposed simulation approach is described in detail. Section II briefly reviews the basics on reversible functions and circuits. Section III considers complete simulation and sketches the general idea of our contribution followed by Section IV describing the precise implementation. The complexity of the solutions are discussed in Section $\mathrm{V}$ and experimentally evaluated in Section VI. Finally, the paper is concluded in Section VII. 
TABLE I

BOOLEAN FUNCTIONS

(a) Irreversible (Adder)

\begin{tabular}{ccc|cc}
$x_{1}$ & $x_{2}$ & $x_{3}$ & $f_{1}$ & $f_{2}$ \\
\hline 0 & 0 & 0 & 0 & 0 \\
0 & 0 & 1 & 0 & 1 \\
0 & 1 & 0 & 0 & 1 \\
0 & 1 & 1 & 1 & 0 \\
1 & 0 & 0 & 0 & 1 \\
1 & 0 & 1 & 1 & 0 \\
1 & 1 & 0 & 1 & 0 \\
1 & 1 & 1 & 1 & 1
\end{tabular}

(b) Irreversible

\begin{tabular}{ccc|ccc}
$x_{1}$ & $x_{2}$ & $x_{3}$ & $f_{1}$ & $f_{2}$ & $f_{3}$ \\
\hline 0 & 0 & 0 & 0 & 0 & 0 \\
0 & 0 & 1 & 0 & 0 & 0 \\
0 & 1 & 0 & 0 & 1 & 0 \\
0 & 1 & 1 & 0 & 1 & 1 \\
1 & 0 & 0 & 1 & 0 & 0 \\
1 & 0 & 1 & 1 & 0 & 1 \\
1 & 1 & 0 & 1 & 1 & 1 \\
1 & 1 & 1 & 1 & 1 & 0
\end{tabular}

(c) Reversible

\begin{tabular}{ccc|ccc}
$x_{1}$ & $x_{2}$ & $x_{3}$ & $f_{1}$ & $f_{2}$ & $f_{3}$ \\
\hline 0 & 0 & 0 & 0 & 0 & 0 \\
0 & 0 & 1 & 0 & 1 & 0 \\
0 & 1 & 0 & 1 & 0 & 0 \\
0 & 1 & 1 & 1 & 0 & 1 \\
1 & 0 & 0 & 0 & 0 & 1 \\
1 & 0 & 1 & 0 & 1 & 1 \\
1 & 1 & 0 & 1 & 1 & 0 \\
1 & 1 & 1 & 1 & 1 & 1
\end{tabular}

\section{REVERSIBLE FUNCTIONS AND CIRCUITS}

Logic computations can be defined as a function over Boolean variables. More precisely:

Definition 1. A Boolean function is a mapping $f: \mathbb{B}^{n} \rightarrow \mathbb{B}$ with $n \in \mathbb{N}$. A function $f$ is defined over its primary input variables $X=\left\{x_{1}, x_{2}, \ldots, x_{n}\right\}$ and hence is also denoted by $f\left(x_{1}, x_{2}, \ldots, x_{n}\right)$. The precise mapping is described in terms of Boolean expressions which are formed over the variables from $X$ and operations like $\wedge$ (AND), $\vee(\mathrm{OR})$, or $\cdot$ (NOT).

A multi-output Boolean function is a mapping $f: \mathbb{B}^{n} \rightarrow \mathbb{B}^{m}$ with $n, m \in \mathbb{N}$. More precisely, it is a system of Boolean functions $f_{i}\left(x_{1}, x_{2}, \ldots, x_{n}\right)$ with $1 \leq i \leq m$. The respective functions $f_{i}$ are also denoted as primary outputs.

Multi-output functions are also denoted as $n$-input, $m$-output functions or $n \times m$ functions, respectively. In this work, realizations of reversible functions are considered. Reversible functions are a subset of multi-output functions and are defined as follows:

Definition 2. A multi-output function $f: \mathbb{B}^{n} \rightarrow \mathbb{B}^{m}$ is reversible iff

- its number of inputs is equal to the number of outputs (i.e. $n=m$ ) and

- it maps each input pattern to a unique output pattern.

In other words, each reversible function is a bijection that performs a permutation of the set of input patterns. A function that is not reversible is termed irreversible.

Example 1. Table I(a) shows the truth table of a 3-input, 2-output function representing a 1-bit adder. This function is irreversible, since $n \neq m$. Also the function in Table $I(b)$ is irreversible. Here, the number $n$ of inputs indeed is equal to the number $m$ of outputs, but there is no unique input-output mapping (e.g. both inputs 000 and 001 map to the output 000). In contrast, the $3 \times 3$ function shown in Table I(c) is reversible, since each input pattern maps to a unique output pattern.

Reversible functions are realized through reversible circuits.

Definition 3. A reversible circuit $G$ is a cascade of reversible gates $G=g_{1} \ldots g_{d}$, where fanout and feedback are not directly allowed [6] and $d$ denotes the number of gates. A reversible gate has the form $g(C, T)$, where $C=\left\{x_{i_{1}}, \ldots, x_{i_{k}}\right\} \subset X$ is the set of control lines and $T=\left\{x_{j_{1}}, \ldots, x_{j_{l}}\right\} \subset X$ with $C \cap T=\emptyset$ is the set of target lines. The set $C$ may be empty.

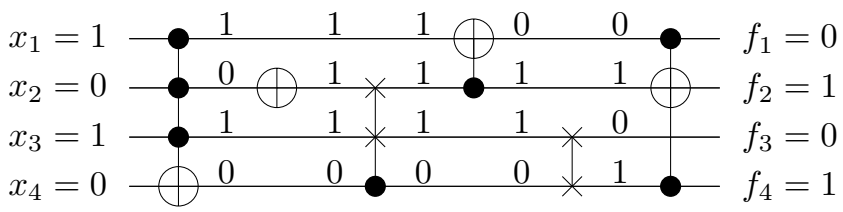

Fig. 1. A reversible circuit

Commonly used reversible gates are:

- The Toffoli gate $\operatorname{TOF}\left(C,\left\{x_{t}\right\}\right)$ [22] which consists of a single target line $x_{t} \in X \backslash C$ whose value is inverted if all values on the control lines are set to 1 or if $C=\emptyset$. All remaining values are passed through the gate unaltered.

- The Fredkin gate $F\left(C,\left\{x_{t 1}, x_{t 2}\right\}\right)$ [23] which consists of two target lines $x_{t 1}, x_{t 2} \in X \backslash C$ interchanges the values of these target lines if all values on the control lines are set to 1 or if $C=\emptyset$, respectively. Again, all remaining values are passed through the gate unaltered.

Example 2. Fig. 1 shows a reversible circuit composed of six reversible gates. Black circles denote the control lines, while the $\oplus$-symbol and the $\times$-symbol is used to denote the target line of the Toffoli gate and the Fredkin gate, respectively. The annotated values demonstrate the computation in the respective gates.

\section{Complete Simulation of Reversible Circuits}

In this work, we consider the complete simulation of reversible circuits, i.e. the determination of all possible output patterns obtained by applying all possible input patterns. For this purpose, we first discuss the straight-forward solution which probably is mostly used in the respective applications relying on simulation. Afterwards, we illustrate the general idea of our improved approach. The precise algorithm is then presented in the next section.

\section{A. Standard Solution}

The straight-forward solution for simulation is rather simple: All input patterns are separately applied to the circuit. Then, for each input pattern, the respective cascades of gates is successively applied eventually leading to the desired output pattern. This complies with the application of the pattern 1010 as illustrated above in Fig. 1. 
TABLE II

ILLUSTRATION OF THE GENERAL IDEA

\begin{tabular}{|c|c|}
\hline \multicolumn{2}{|c|}{ (a) Identity } \\
\hline Inp. & Out. \\
\hline$a b c d$ & $a b c d$ \\
\hline $0 \begin{array}{llll}0 & 0 & 0 & 0\end{array}$ & $\begin{array}{lllll}0 & 0 & 0 & 0\end{array}$ \\
\hline $\begin{array}{lllll}0 & 0 & 0 & 1\end{array}$ & $\begin{array}{lllll}0 & 0 & 0 & 1\end{array}$ \\
\hline $\begin{array}{llll}0 & 0 & 1 & 0\end{array}$ & $\begin{array}{llll}0 & 0 & 1 & 0\end{array}$ \\
\hline $\begin{array}{lllll}0 & 0 & 1 & 1\end{array}$ & $\begin{array}{lllll}0 & 0 & 1 & 1\end{array}$ \\
\hline $\begin{array}{llll}0 & 1 & 0 & 0\end{array}$ & $\begin{array}{llll}0 & 1 & 0 & 0\end{array}$ \\
\hline $\begin{array}{lllll}0 & 1 & 0 & 1\end{array}$ & $\begin{array}{llll}0 & 1 & 0 & 1\end{array}$ \\
\hline $\begin{array}{llll}0 & 1 & 1 & 0\end{array}$ & $\begin{array}{lllllll}0 & 1 & 1 & 0\end{array}$ \\
\hline $\begin{array}{lllll}0 & 1 & 1 & 1\end{array}$ & $\begin{array}{lllll}0 & 1 & 1 & 1\end{array}$ \\
\hline 1000 & 1000 \\
\hline $\begin{array}{llll}1 & 0 & 0 & 1\end{array}$ & $\begin{array}{llll}1 & 0 & 0 & 1\end{array}$ \\
\hline $\begin{array}{llll}10 & 10\end{array}$ & 1010 \\
\hline 1011 & $\begin{array}{llll}1 & 0 & 1 & 1\end{array}$ \\
\hline 1100 & 1100 \\
\hline $\begin{array}{llll}1 & 1 & 0 & 1\end{array}$ & $\begin{array}{llll}1 & 1 & 0 & 1\end{array}$ \\
\hline 1110 & 1110 \\
\hline & $\begin{array}{llll}1 & 1 & 1 & 1\end{array}$ \\
\hline
\end{tabular}

\begin{tabular}{|c|c|}
\hline \multicolumn{2}{|c|}{ (c) $2^{\text {nd }}$ Gate } \\
\hline Inp. & Out. \\
\hline$a b c d$ & $a b c d$ \\
\hline $\begin{array}{lllllll} & 0 & 0 & 0\end{array}$ & 0100 \\
\hline 001 & 10 \\
\hline 0 & 01 \\
\hline 011 & \\
\hline & \\
\hline & \\
\hline 110 & $\begin{array}{llll}0 & 0 & 1\end{array}$ \\
\hline 111 & \\
\hline & \\
\hline 01 & 1110 \\
\hline 1010 & 111 \\
\hline & \\
\hline & \\
\hline 11 & 100 \\
\hline 1110 & $\begin{array}{lll}101 & 1\end{array}$ \\
\hline & \\
\hline
\end{tabular}

\begin{tabular}{|c|c|}
\hline \multicolumn{2}{|c|}{ (d) $3^{r d}$ Gate } \\
\hline Inp. & Out. \\
\hline $\mathrm{abcd}$ & $a b c d$ \\
\hline 00000 & $\begin{array}{llllll} & 1 & 1 & 0 & 0\end{array}$ \\
\hline $\begin{array}{lll}0 & 0 & 1\end{array}$ & $\begin{array}{lllll}0 & 0 & 1\end{array}$ \\
\hline 10 & 01 \\
\hline & \\
\hline & \\
\hline 101 & \\
\hline 110 & $\begin{array}{llll}0 & 0 & 1 & 0\end{array}$ \\
\hline 111 & $\begin{array}{llll}0 & 1 & 0\end{array}$ \\
\hline & \\
\hline $\begin{array}{llll}0 & 01\end{array}$ & $\begin{array}{llll}1 & 0 & 1 & 1\end{array}$ \\
\hline 1010 & $\begin{array}{llll}1 & 1 & 1 & 0\end{array}$ \\
\hline 1011 & 1111 \\
\hline 1 & \\
\hline 110 & $\begin{array}{llll}1 & 0 & 0 & 1\end{array}$ \\
\hline 1110 & $\begin{array}{llll}1 & 1 & 0 & 1\end{array}$ \\
\hline & \\
\hline
\end{tabular}

(e) $4^{\text {th }}$ Gate

Inp. Out.

\begin{tabular}{l|l}
$\mathrm{abcd} a \mathrm{acd}$ \\
\hline 0000
\end{tabular}

$\begin{array}{lllllllllllll}0 & 0 & 0 & 1 & 0 & 0 & 1 & 1\end{array}$

$\begin{array}{lllllllll}0 & 0 & 1 & 0 & \mathbf{1} & 1 & 1 & \mathbf{0}\end{array}$

\begin{tabular}{llll|llll}
0 & 0 & 1 & 1 & 1 & 1 & 1 & 1
\end{tabular}

\begin{tabular}{llll|llllllllllllllll}
0 & 1 & 0 & 0 & 0 & 0 & 0 & 0
\end{tabular}

\begin{tabular}{lllll|llll}
0 & 1 & 0 & 1 & 0 & 0 & 0 & 1
\end{tabular}

\begin{tabular}{llll|llllllllll}
0 & 1 & 1 & 0 & 0 & 0 & 1 & 0
\end{tabular}

$\begin{array}{lllllllll}0 & 1 & 1 & 1 & \mathbf{1} & \mathbf{1} & \mathbf{0} & \mathbf{1}\end{array}$

$\begin{array}{llllllll}1 & 0 & 0 & 0 & \mathbf{0} & \mathbf{1} & \mathbf{0} & \mathbf{0}\end{array}$

$\begin{array}{lllllllll}1 & 0 & 0 & 1 & 1 & 0 & 1 & 1\end{array}$

$\begin{array}{lllllllll}1 & 0 & 1 & 0 & \mathbf{0} & 1 & 1 & 0\end{array}$

\begin{tabular}{llll|llll}
1 & 0 & 1 & 1 & $\mathbf{0}$ & 1 & 1 & 1
\end{tabular}

$\begin{array}{lllllllllllllll}1 & 1 & 0 & 0 & 1 & 0 & 0 & 0\end{array}$

$\begin{array}{lllllllll}1 & 1 & 0 & 1 & 1 & 0 & 0 & 1\end{array}$

$\begin{array}{llllllll}1 & 1 & 1 & 0 & \mathbf{O} & 1 & 0 & 1\end{array}$

\begin{tabular}{llll|llll}
1 & 1 & 1 & 1 & 1 & 0 & 1 & 0
\end{tabular}

\begin{tabular}{|c|c|}
\hline \multicolumn{2}{|c|}{ (f) $5^{\text {th }}$ Gate } \\
\hline Inp. & Out. \\
\hline $\mathrm{abcc}$ & $a b c d$ \\
\hline$\overline{0000}$ & $\begin{array}{llll}1 & 1 & 0 & 0\end{array}$ \\
\hline $\begin{array}{llll}0 & 0 & 1\end{array}$ & $\begin{array}{llll}0 & 0 & 1\end{array}$ \\
\hline 010 & 110 \\
\hline 01011 & \\
\hline 100 & \\
\hline 101 & $\begin{array}{lllll}0 & 0 & 1\end{array}$ \\
\hline 110 & $\begin{array}{llll}0 & 0 & 0\end{array}$ \\
\hline 1 & 1 \\
\hline & \\
\hline 1001 & $\begin{array}{lll}10 & 1\end{array}$ \\
\hline 1010 & 010 \\
\hline 10 & \\
\hline 11 & 100 \\
\hline 11 & 101 \\
\hline 1110 & $\begin{array}{lllll}0 & 1 & 1 & 0\end{array}$ \\
\hline & \\
\hline
\end{tabular}

(g) $6^{\text {th }}$ Gate

\begin{tabular}{c|c} 
Inp. & Out. \\
\hline $\mathrm{abc} \mathrm{d}$ & $\mathrm{a} \mathrm{b} \mathrm{c} \mathrm{d}$
\end{tabular}

00000011100

$\begin{array}{lllllllll}0 & 0 & 0 & 1 & 0 & 0 & 1 & 1\end{array}$

$\begin{array}{lllllllll}0 & 0 & 1 & 0 & \mathbf{1} & \mathbf{0} & \mathbf{0} & 1\end{array}$

$\begin{array}{llllllll}0 & 0 & 1 & 1 & \mathbf{1} & \mathbf{0} & 1 & \mathbf{1}\end{array}$

$\begin{array}{llllllllllllllll}0 & 1 & 0 & 0 & 0 & 0 & 0 & 0\end{array}$

\begin{tabular}{llll|lllllllllll}
0 & 1 & 0 & 1 & 0 & 0 & 1 & 0
\end{tabular}

$\begin{array}{llllllllllll}0 & 1 & 1 & 0 & 0 & 0 & 0 & 1\end{array}$

$\begin{array}{llllllllllllllll}0 & 1 & 1 & 1 & 1 & 1 & 1 & 0\end{array}$

$\begin{array}{llllllllllllllll}1 & 0 & 0 & 0 & 0 & 1 & 0 & 0\end{array}$

$\begin{array}{llllllll}1 & 0 & 0 & 1 & 1 & 1 & 1 & 1\end{array}$

\begin{tabular}{llll|lllll}
1 & 0 & 1 & 0 & 0 & 1 & 0 & 1
\end{tabular}

$\begin{array}{llllllll}1 & 0 & 1 & 1 & 0 & 1 & 1 & 1\end{array}$

$\begin{array}{lllllllllllll}1 & 1 & 0 & 0 & 1 & 0 & 0 & 0\end{array}$

$\begin{array}{llllllllllllll}1 & 1 & 0 & 1 & 1 & 0 & 1 & 0\end{array}$

\begin{tabular}{llll|lllllll}
1 & 1 & 1 & 0 & 0 & 1 & 1 & 0
\end{tabular}

\begin{tabular}{llll|llll}
1 & 1 & 1 & 1 & 1 & 1 & 0 & 1
\end{tabular}
Because of this simplicity, this solution is heavily used in many applications such as validation, optimization, automatic test pattern generation, or debugging of reversible circuits. In total, this leads to a run-time complexity of $2^{n} \cdot d$ with $n$ and $d$ being the number of circuit lines and gates, respectively.

\section{B. General Idea for Improvement}

Considering reversible circuits, complete simulation can significantly been improved by exploiting two certain characteristics of this type of computation:

1) Functional Effect of Single Gates

For many input patterns, single gates often have no effect, i.e. often just very small sets of inputs are actually leading to different output patterns. In fact, e.g. a Toffoli gate $g(C, T)$ only has an effect for the $2^{n-|C|}$ input patterns where all control lines $x \in C$ are set to 1 .

2) Reversible Gates Realize Permutations

Reversible gates always realize a permutation of two input patterns, i.e. if e.g. an input pattern 0000 maps to an output pattern 0001, then the input pattern 0001 must map to an output pattern 0000, too. By this, each gate eventually leads to an exchange of input patterns.

Example 3. Consider again the circuit from Fig. 1. Considering all $2^{4}=16$ possible input patterns for this circuit, then

- gate $g_{1}$ modifies only 2 of them,

- gate $g_{2}$ modifies all 16 of them,

- gate $g_{3}$ modifies only 4 of them,

- gate $g_{4}$ modifies only 8 of them,

- gate $g_{5}$ modifies all 8 of them, and

- gate $g_{6}$ modifies only 4 of them.

Furthermore, e.g. the first gate just swaps the respective patterns 1110 and 1111. The same holds e.g. for the sixth gate where all patterns of the form $11-1$ are swapped with the corresponding pattern of the form $10-1$.

These characteristics enable a complete simulation without the need for the application of $2^{n} \cdot d$ steps. In fact, in order to simulate e.g. the first gate from Fig. 1, just two steps (for 1110 and 1111) are necessary. The remaining 14 simulations steps can be omitted because, due to the structure of the gate, they have no affect on the result. Moreover, this number of steps can be reduced further by exploiting the second characteristic: Since each gate has a unique input/output mapping, the corresponding computation just leads to an exchange of patterns. For example, the first gate from Fig. 1 simply swaps 1110 and 1110 . Because of this, it is sufficient to only consider patterns with a target line set to 1 (in this case 1111) ${ }^{1}$. This reduces the number of patterns to be considered to just a single one (instead of 16 when the standard solution is applied).

This is exploited in the proposed simulation approach. Instead of separately applying all possible input patterns, we suggest a methodology that considers all patterns at once. Then, the application of a single gate does not consider each pattern separately, but just performs the respective swaps on exactly those patterns which are actually affected by the gate. The configuration of these affected patterns can easily be determined from the type and the set of control lines of the considered gate.

The application of this idea is illustrated by the following example.

Example 4. Following the general idea from above, the circuit depicted in Fig. 1 shall completely been simulated. The respective steps are given in Table II. We start with a representation of all $2^{4}=16$ possible pattern. As long as no gate is applied, the identity results (see Table II(a)). Then, the respective gates are applied.

As discussed above, the first gate in Fig. 1 only swaps the patterns 1110 and 1111. That is, only one step has to be performed to simulate this gate (leading to Table II $(b)$ ). The second gate is the worst case as all patterns are affected. Nevertheless, still only half the steps compared to standard simulation are necessary since each pattern of the form -1-is simply swapped with the corresponding pattern of the form -0-- (leading to Table II(c)). The third gate represents a Fredkin gate. Because of the control line, this shall affect patterns of the form - - - However, patterns with both

\footnotetext{
${ }^{1}$ In a similar fashion, this also holds for the Fredkin gate.
} 


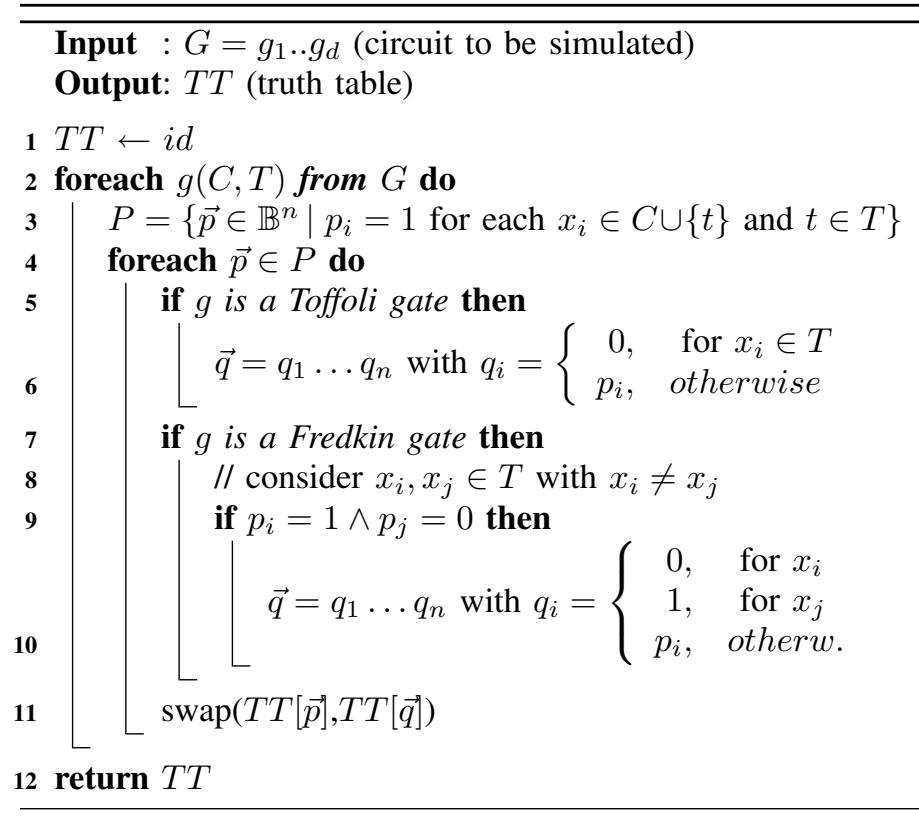

Fig. 2. Proposed simulation algorithm

target lines set to the same value are also not affected (as Fredkin gates swap the value of the target lines). Hence, for this gate only the patterns 0011 and 1011 are swapped with 0101 and 1101, respectively (leading to Table II(d)). In a similar fashion all remaining gates are applied eventually leading to the results of the complete simulation as shown in Table II $(g)$.

\section{IMPLEMENTATION}

Motivated by the general idea sketched above, this section presents the improved algorithm for complete simulation of reversible circuits. The algorithm does not simulate single patterns separately, but considers all of them at once. The execution of gates is conducted by swapping those patterns which are actually affected by the currently considered gate. The configuration of these affected patterns is determined from the type and the set of control lines of the considered gate. More precisely:

Definition 4. Let $g(C, T)$ be a reversible gate in a circuit over $n$ lines and the inputs $X=\left\{x_{1} \ldots x_{n}\right\}$. All patterns with the respective control line set to 1 are affected by this gate. Furthermore, due to the permutative nature of reversible gates, only those pattern with their first target line set to 1 have to be considered. Hence, the patterns to be considered can be summarized in a set $P$ composed of all patterns of the form $\vec{p} \in \mathbb{B}^{n}$ with $p_{i}=1$ for each $x_{i} \in C \cup\{t\}$ with $t \in T$ and $i \in\{1 \ldots n\}$.

Example 5. Consider the last gate of the circuit from Fig. 1. The corresponding set $M$ of patterns to be considered is $P=\{1101,1111\}$.

Having this definition, the proposed simulation approach follows the algorithm as sketched in Fig. 2. This algorithm gets a circuit $G$ to be simulated and generates the complete function (in terms of a truth table) realized by $G$. In the following, the respective steps are discussed.

First, the truth table to be generated is initialized with the identity function (Line 1). Then, each gate $g(C, T)$ of the circuit $G$ is traversed from the outputs to the inputs (Line 2). In each iteration, the set $P$ of patterns to be considered for the current gate $g$ is created according to Definition 4 (Line 3). For each of those patterns $\vec{p} \in P$ the corresponding counterpart $\vec{q}$, i.e. the pattern to be swapped with $\vec{p}$, is created next (Line 410). This depends on the type of the current gate $g$ :

- In case of a Toffoli gate (Line 5/6), an inversion is conducted at the target line. Hence, $\vec{q}$ is equal to $\vec{p}$ except for the target line which is set to 0 (instead of 1).

- In case of a Fredkin gate (Line 7-10), the value of one of the target lines (1, in the currently considered pattern) is swapped with the value of the other target line. This only has an effect, if the other target line is 0 . Hence, only patterns $\vec{p}$ with $p_{i}=1$ and $p_{j}=0$ for $x_{i}, x_{j} \in T$ and $x_{i} \neq x_{j}$ are considered. The counterpart $q$ is then defined by the opposite assignment of the target lines.

By this, an affected pattern $\vec{p}$ and its corresponding counterpart $\vec{q}$ have been determined and, thus, only need to be swapped in the truth table representation (Line 11). If all affected patterns and all gates have been considered accordingly, the algorithm returns the resulting truth table and terminates (Line 12).

\section{DisCusSION}

Following the procedure described above leads to an improved run-time complexity in comparison to the standard simulation. In fact, we can distinguish between a best case and a worst case:

- The best case can be observed when the circuit is solely composed of Toffoli gates with the maximum number of control lines (i.e. of Toffoli gates $g(C, T)$ with $|C|=n-1)$. Then, the simulation of each gate just requires one general step, namely swapping two patterns. This leads to $d$ steps for the whole circuit. As the initial creation of all possible patterns requires $2^{n}$ steps, we eventually get $2^{n}+d$ steps in the best case. Compared to the standard approach with its $2^{n} \cdot d$ steps, this is considerably smaller. The number of gates hardly affects the total run-time anymore.

- The worst case can be observed when the circuit is solely composed of Toffoli gates with no control lines at all (i.e. of Toffoli gates $g(C, T)$ with $C=\emptyset$ ). Then, all patterns are affected. However, due to the exploitation of the permutation characteristic, still only half the swapping steps need to be considered. This eventually leads to $2^{n}+\frac{\left(2^{n} \cdot d\right)}{2}$ steps in the worst case. But since circuits solely composed of Toffoli gates with no control lines (i.e. basically NOT gates) hardly occur, this worst case does not occur frequently.

In both cases, the complexity is considerably less compared to the standard simulation. Of course, the major bottleneck remains the exponential complexity. However, as simulation still is heavily applied in various applications for small circuits 
(where exponential complexity is feasible), the improvements enabled by the proposed simulation approach are beneficial. This is also confirmed by actual run-time measurements conducted in our experimental evaluation which is summarized in the next section.

\section{EXPERIMENTAL EVALUATION}

The approach introduced in Section IV has been implemented in C++ on top of RevKit [24]. In order to evaluate the efficiency of the approach, the proposed solution has been applied to completely simulate a wide range of benchmarks circuits taken from RevLib [25]. Then, the resulting run-time has been compared to the corresponding results obtained using a standard simulation ${ }^{2}$. All experiments have been conducted on an Intel Core i5-2430M machine with $2.4 \mathrm{GHz}$ and $6 \mathrm{~GB}$ of memory running Linux.

Table III summarizes the results. The first columns provide the name (denoted by Circuit), the number of lines (denoted by $n$ ), and the number of gates (denoted by $d$ ) of the respectively considered benchmark circuits. The runtime (in milliseconds) required for standard simulation and the proposed simulation scheme are given in the columns denoted by Standard and Proposed, respectively. The improvement of the proposed approach, i.e. the run-time of the standard approach divided by the run-time of the proposed simulation, is provided in Column Impr.

The results clearly confirm the benefits of the proposed approach. For all benchmark circuits, significant reductions in the needed run-time can be observed. In particular for circuits with a large number of gates, the increase in the performance is evident. In these cases improvements of more than three orders of magnitude can be observed. Instead of waiting several minutes for a result, the proposed approach delivers the results in a fraction of a second. In particular, for approaches which rely on frequent complete simulation runs (such as window optimization [13]), this speed-up is essential.

Besides that, Table III also provides some numbers concerning the complexity discussion from Section V. As discussed there, the proposed simulation approach has a worst case and best case complexity of $2^{n}+\frac{\left(2^{n} \cdot d\right)}{2}$ and $2^{n}+d$, respectively, compared to the complexity of the standard simulation (which is $2^{n} \cdot d$ ). Applied to precise benchmark circuits, Columns Complexity Impr. of Table III show the improvements with respect to the worst case (denoted by $w c$ ) and the best case (denoted by $b c$ ). Also these numbers confirm the discussions from above. For almost all benchmark circuits, the actual run-time improvement (Column Impr.) indeed is between the estimated worst and best case; in most of the cases a bit closer to the worst case.

Overall, the proposed algorithm provides an alternative simulation method that is as easy to implement as the standard simulation, but has significant benefits with respect to the resulting complexity which has also been confirmed by the actual application to a large set of circuits.

\footnotetext{
${ }^{2}$ In fact, we used the open source simulation engine provided in RevKit [24]
}

\section{CONCLUSION}

In this paper, we introduced an approach for complete simulation of reversible circuits which explicitly exploited the reversibility of the underlying computation paradigm. In particular, we utilized the fact that (1) usually just a very small set of patterns is affected by a gate and (2) that gates simply lead to an exchange of input patterns. Although the proposed solution still remains exponential in the complexity, improvements of up to three orders of magnitude can be achieved compared to the standard simulation. Nevertheless, due to the complexity the approach still is restricted to circuits with a small number of inputs/outputs only. Furthermore, the approach only supports complete simulation thus far. However, for all applications which rely on frequent complete simulation runs (such as window optimization [13]) the proposed methods offers a simple but effective alternative.

\section{REFERENCES}

[1] R. Landauer, "Irreversibility and heat generation in the computing process," IBM J. Res. Dev., vol. 5, p. 183, 1961.

[2] C. H. Bennett, "Logical reversibility of computation," IBM J. Res. Dev, vol. 17 , no. 6, pp. 525-532, 1973.

[3] B. Desoete and A. D. Vos, "A reversible carry-look-ahead adder using control gates," INTEGRATION, the VLSI Jour, vol. 33, no. 1-2, pp. 89-104, 2002.

[4] P. Patra and D. Fussell, "On efficient adiabatic design of MOS circuits," in Workshop on Physics and Computation, Boston, 1996, pp. 260-269.

[5] R. Wille, R. Drechsler, C. Oswald, and A. Garcia-Ortiz, "Automatic design of low-power encoders using reversible circuit synthesis," in Design, Automation and Test in Europe, 2012, pp. 1036-1041.

[6] M. Nielsen and I. Chuang, Quantum Computation and Quantum Information. Cambridge Univ. Press, 2000.

[7] R. Glück and M. Kawabe, "A method for automatic program inversion based on LR(0) parsing," Fundamenta Informaticae, vol. 66, no. 4, pp. 367-395, 012005.

[8] D. M. Miller, D. Maslov, and G. W. Dueck, "A transformation based algorithm for reversible logic synthesis," in Design Automation Conf., 2003, pp. 318-323.

[9] V. V. Shende, A. K. Prasad, I. L. Markov, and J. P. Hayes, "Synthesis of reversible logic circuits," IEEE Trans. on CAD, vol. 22, no. 6, pp 710-722, 2003.

[10] R. Wille and R. Drechsler, "BDD-based synthesis of reversible logic for large functions," in Design Automation Conf., 2009, pp. 270-275.

[11] M. Soeken, R. Wille, C. Hilken, N. Przigoda, and R. Drechsler, "Synthesis of reversible circuits with minimal lines for large functions," in ASP Design Automation Conf., 2012, pp. 85-92.

[12] D. Y. Feinstein, M. A. Thornton, and D. M. Miller, "Partially redundant logic detection using symbolic equivalence checking in reversible and irreversible logic circuits," in Design, Automation and Test in Europe, 2008, pp. 1378-1381.

[13] M. Soeken, R. Wille, G. W. Dueck, and R. Drechsler, "Window optimization of reversible and quantum circuits," in Symposium on Design and Diagnostics of Electronic Circuits and Systems, 2010, pp. 341-345.

[14] G. F. Viamontes, I. L. Markov, and J. P. Hayes, "Checking equivalence of quantum circuits and states," in Int'l Conf. on CAD, 2007, pp. 69-74.

[15] S.-A. Wang, C.-Y. Lu, I.-M. Tsai, and S.-Y. Kuo, "An XQDD-based verification method for quantum circuits," IEICE Transactions, vol. 91A, no. 2, pp. 584-594, 2008.

[16] R. Wille, D. Große, S. Frehse, G. W. Dueck, and R. Drechsler, "Debugging of Toffoli networks," in Design, Automation and Test in Europe, 2009, pp. 1284-1289.

[17] I. Polian, T. Fiehn, B. Becker, and J. P. Hayes, "A family of logical fault models for reversible circuits," in Asian Test Symp., 2005, pp. 422-427.

[18] R. Wille, H. Zhang, and R. Drechsler, "ATPG for reversible circuits using simulation, Boolean satisfiability, and pseudo Boolean optimization," in IEEE Annual Symposium on VLSI, 2011, pp. 120-125.

[19] R. Drechsler and R. Wille, "From truth tables to programming languages: Progress in the design of reversible circuits," in Int'l Symp. on Multi-Valued Logic, 2011, pp. 78-85. 
TABLE III

EXPERIMENTAL EVALUATION

\begin{tabular}{|c|c|c|c|c|c|c|c|}
\hline \multirow[b]{2}{*}{ Circuit } & \multirow[b]{2}{*}{$n$} & \multirow[b]{2}{*}{$d$} & \multicolumn{3}{|c|}{ Simulation Run-time } & \multicolumn{2}{|c|}{ Complexity Impr. } \\
\hline & & & Standard & Proposed & Impr. & wc & bc \\
\hline urf6 160 & 15 & 10740 & 663459 & 1639 & 404.8 & 2.0 & 8089.6 \\
\hline urf6_281 & 15 & 5088 & 349400 & 2021 & 172.9 & 2.0 & 4405.0 \\
\hline urf4_187 & 11 & 32004 & 152443 & 279 & 545.4 & 2.0 & 1924.9 \\
\hline alu3_200 & 18 & 94 & 63286 & 12458 & 5.1 & 2.0 & 95.0 \\
\hline urf3_155 & 10 & 26468 & 52481 & 142 & 369.9 & 2.0 & 985.9 \\
\hline sqr6_259 & 18 & 81 & 52346 & 13335 & 3.9 & 2.0 & 82.0 \\
\hline 5xp1_194 & 17 & 85 & 28241 & 5998 & 4.7 & 2.0 & 85.9 \\
\hline dk27_225 & 18 & 24 & 24266 & 12470 & 1.9 & 1.9 & 25.0 \\
\hline urf3_279 & 10 & 14075 & 24174 & 95 & 254.4 & 2.0 & 954.6 \\
\hline example2_231 & 16 & 157 & 23937 & 2586 & 9.3 & 2.0 & 157.6 \\
\hline alu2_199 & 16 & 157 & 23682 & 2594 & 9.1 & 2.0 & 157.6 \\
\hline ryy6_256 & 17 & 44 & 23389 & 5898 & 4.0 & 2.0 & 45.0 \\
\hline mlp4_245 & 16 & 131 & 21064 & 2687 & 7.8 & 2.0 & 131.7 \\
\hline x2_267 & 17 & 38 & 16692 & 6970 & 2.4 & 2.0 & 39.0 \\
\hline inc_237 & 16 & 93 & 15635 & 2598 & 6.0 & 2.0 & 93.9 \\
\hline parity_247 & 17 & 32 & 13148 & 5991 & 2.2 & 1.9 & 33.0 \\
\hline urf1_149 & 9 & 11554 & 11520 & 58 & 197.1 & 2.0 & 490.3 \\
\hline t481_263 & 17 & 21 & 11406 & 6001 & 1.9 & 1.9 & 22.0 \\
\hline ham15_107 & 15 & 132 & 9991 & 1242 & 8.0 & 2.0 & 132.5 \\
\hline urf5_158 & 9 & 10276 & 9882 & 53 & 186.9 & 2.0 & 487.7 \\
\hline urf3_156 & 10 & 2732 & 7826 & 41 & 191.2 & 2.0 & 745.1 \\
\hline clip_206 & 14 & 174 & 7603 & 587 & 12.9 & 2.0 & 173.2 \\
\hline ham15_109 & 15 & 109 & 7551 & 1482 & 5.1 & 2.0 & 109.6 \\
\hline dc2_222 & 15 & 75 & 7129 & 1240 & 5.7 & 2.0 & 75.8 \\
\hline cnt3-5_179 & 16 & 25 & 6697 & 2608 & 2.6 & 1.9 & 26.0 \\
\hline ham15_108 & 15 & 70 & 6617 & 1601 & 4.1 & 2.0 & 70.8 \\
\hline urf3_157 & 10 & 2674 & 6613 & 39 & 168.8 & 2.0 & 740.7 \\
\hline urf1_278 & 9 & 6761 & 5967 & 40 & 147.8 & 2.0 & 476.0 \\
\hline cnt3-5_180 & 16 & 20 & 5480 & 2622 & 2.1 & 1.9 & 21.0 \\
\hline misex1_241 & 15 & 55 & 5110 & 1219 & 4.2 & 2.0 & 55.9 \\
\hline co14_215 & 15 & 30 & 4692 & 1307 & 3.6 & 1.9 & 31.0 \\
\hline urf5_280 & 9 & 5097 & 4638 & 39 & 120.1 & 2.0 & 465.4 \\
\hline sao2 257 & 14 & 88 & 4076 & 546 & 7.5 & 2.0 & 88.5 \\
\hline rd84_142 & 15 & 28 & 3571 & 1332 & 2.7 & 1.9 & 29.0 \\
\hline dist_223 & 13 & 185 & 3535 & 248 & 14.3 & 2.0 & 181.9 \\
\hline cm85a_209 & 14 & 69 & 3407 & 784 & 4.3 & 2.0 & 69.7 \\
\hline urf2_152 & 8 & 5030 & 2440 & 22 & 109.5 & 2.0 & 243.7 \\
\hline cm42a_207 & 14 & 35 & 2070 & 712 & 2.9 & 1.9 & 35.9 \\
\hline pm1_249 & 14 & 35 & 2053 & 698 & 2.9 & 1.9 & 35.9 \\
\hline hwb9_123 & 9 & 1959 & 1975 & 26 & 77.1 & 2.0 & 406.1 \\
\hline root 255 & 13 & 99 & 1960 & 258 & 7.6 & 2.0 & 98.8 \\
\hline 0410184_169 & 14 & 46 & 1893 & 559 & 3.4 & 2.0 & 46.9 \\
\hline hwb9_121 & 9 & 1541 & 1800 & 23 & 79.6 & 2.0 & 384.6 \\
\hline urf1_150 & 9 & 1517 & 1797 & 19 & 92.7 & 2.0 & 383.1 \\
\hline hwb9_119 & 9 & 1544 & 1785 & 23 & 78.4 & 2.0 & 384.7 \\
\hline urf1_-̄151 & 9 & 1487 & 1763 & 21 & 83.9 & 2.0 & 381.1 \\
\hline sym6_316 & 14 & 29 & 1722 & 576 & 3.0 & 1.9 & 29.9 \\
\hline urf2_161 & 8 & 3250 & 1639 & 17 & 99.3 & 2.0 & 237.4 \\
\hline urf $2 \_277$ & 8 & 3144 & 1590 & 21 & 75.3 & 2.0 & 236.8 \\
\hline radd_250 & 13 & 48 & 1245 & 261 & 4.8 & 2.0 & 48.7 \\
\hline adr4_197 & 13 & 55 & 1133 & 261 & 4.3 & 2.0 & 55.6 \\
\hline squar5_261 & 13 & 43 & 1043 & 254 & 4.1 & 2.0 & 43.8 \\
\hline rd84_253 & 12 & 111 & 995 & 121 & 8.3 & 2.0 & 109.0 \\
\hline sym10_262 & 11 & 194 & 922 & 59 & 15.7 & 2.0 & 178.1 \\
\hline rd53_311 & 13 & 34 & 778 & 259 & 3.0 & 1.9 & 34.9 \\
\hline urf5_159 & 9 & 499 & 631 & 15 & 43.4 & 2.0 & 253.2 \\
\hline sym9_148 & 10 & 210 & 513 & 29 & 17.5 & 2.0 & 175.1 \\
\hline sqrt8_260 & 12 & 40 & 476 & 150 & 3.2 & 2.0 & 40.6 \\
\hline dc1_220 & 11 & 39 & 439 & 91 & 4.8 & 2.0 & 39.3 \\
\hline
\end{tabular}

\begin{tabular}{|c|c|c|c|c|c|c|c|}
\hline \multirow[b]{2}{*}{ Circuit } & \multirow[b]{2}{*}{$n$} & \multirow[b]{2}{*}{$d$} & \multicolumn{3}{|c|}{ Simulation Run-time } & \multicolumn{2}{|c|}{ Complexity Impr. } \\
\hline & & & Standard & Proposed & Impr. & wc & bc \\
\hline dc1_221 & 11 & 39 & 402 & 93 & 4.3 & 2.0 & 39.3 \\
\hline urf2_153 & 8 & 638 & 374 & 8 & 46.8 & 2.0 & 183.0 \\
\hline hwb8_116 & 8 & 749 & 364 & 8 & 45.4 & 2.0 & 191.0 \\
\hline hwb8_113 & 8 & 637 & 363 & 11 & 32.7 & 2.0 & 182.9 \\
\hline urf2_154 & 8 & 620 & 354 & 8 & 45.2 & 2.0 & 181.5 \\
\hline sym9_146 & 12 & 28 & 353 & 121 & 2.9 & 1.9 & 28.8 \\
\hline cycle10_2_110 & 12 & 19 & 349 & 131 & 2.7 & 1.9 & 19.9 \\
\hline hwb8_114 & 8 & 614 & 348 & 8 & 45.7 & 2.0 & 181.0 \\
\hline 9symml_195 & 10 & 129 & 303 & 29 & 10.3 & 2.0 & 115.5 \\
\hline sym9_193 & 10 & 129 & 295 & 36 & 8.3 & 2.0 & 115.5 \\
\hline cm152a_212 & 12 & 16 & 261 & 131 & 2.0 & 1.9 & 16.9 \\
\hline z4ml_269 & 11 & 48 & 258 & 59 & 4.4 & 2.0 & 47.9 \\
\hline $\mathrm{z} 4 \_268$ & 11 & 48 & 255 & 54 & 4.8 & 2.0 & 47.9 \\
\hline life_238 & 10 & 107 & 254 & 27 & 9.5 & 2.0 & 97.8 \\
\hline $\max 46 \_240$ & 10 & 107 & 249 & 35 & 7.1 & 2.0 & 97.8 \\
\hline sqn_258 & 10 & 76 & 190 & 27 & 7.1 & 2.0 & 71.7 \\
\hline rd73_252 & 10 & 80 & 185 & 29 & 6.4 & 2.0 & 75.1 \\
\hline hwb7_59 & 7 & 289 & 170 & 5 & 33.3 & 2.0 & 89.0 \\
\hline wim_266 & 11 & 25 & 155 & 60 & 2.6 & 1.9 & 25.7 \\
\hline rd73_140 & 10 & 20 & 100 & 30 & 3.4 & 1.9 & 20.6 \\
\hline hwb7_62 & 7 & 331 & 84 & 4 & 23.7 & 2.0 & 92.6 \\
\hline sys6-v0_111 & 10 & 20 & 75 & 28 & 2.7 & 1.9 & 20.6 \\
\hline hwb7_61 & 7 & 236 & 71 & 4 & 19.2 & 2.0 & 83.3 \\
\hline mini_alu_305 & 10 & 20 & 69 & 27 & 2.5 & 1.9 & 20.6 \\
\hline hwb7_60 & 7 & 166 & 51 & 4 & 14.0 & 2.0 & 72.7 \\
\hline con1_216 & 9 & 21 & 38 & 12 & 3.2 & 1.9 & 21.1 \\
\hline rd53_251 & 8 & 27 & 21 & 5 & 3.8 & 1.9 & 25.3 \\
\hline hwb6_56 & 6 & 126 & 19 & 2 & 10.6 & 2.0 & 42.8 \\
\hline f2_232 & 8 & 19 & 18 & 5 & 3.8 & 1.9 & 18.6 \\
\hline cm82a_208 & 8 & 22 & 18 & 5 & 3.3 & 1.9 & 21.2 \\
\hline rd53_130 & 7 & 30 & 15 & 4 & 4.2 & 1.9 & 25.1 \\
\hline rd53_138 & 8 & 12 & 15 & 5 & 2.7 & 1.9 & 12.4 \\
\hline sym6_145 & 7 & 36 & 14 & 3 & 4.5 & 1.9 & 28.9 \\
\hline rd53_131 & 7 & 28 & 14 & 5 & 2.9 & 1.9 & 23.8 \\
\hline ham7_104 & 7 & 23 & 10 & 3 & 3.4 & 1.9 & 20.3 \\
\hline hwb6_57 & 6 & 65 & 9 & 2 & 5.6 & 2.0 & 32.7 \\
\hline ham7_106 & 7 & 25 & 9 & 3 & 3.3 & 1.9 & 21.8 \\
\hline ham7_105 & 7 & 21 & 9 & 3 & 3.2 & 1.9 & 18.9 \\
\hline majority_239 & 6 & 8 & 8 & 3 & 2.9 & 1.8 & 8.0 \\
\hline rd53_135 & 7 & 16 & 7 & 3 & 2.6 & 1.9 & 15.1 \\
\hline hwb6_58 & 6 & 42 & 7 & 2 & 4.5 & 2.0 & 26.0 \\
\hline rd53_137 & 7 & 16 & 7 & 3 & 2.5 & 1.9 & 15.1 \\
\hline rd53_133 & 7 & 12 & 6 & 3 & 2.4 & 1.9 & 11.9 \\
\hline C17_204 & 7 & 9 & 6 & 3 & 2.3 & 1.8 & 9.3 \\
\hline alu-bdd_288 & 7 & 9 & 5 & 3 & 1.6 & 1.8 & 9.3 \\
\hline 4mod5-bdd_287 & 7 & 8 & 5 & 3 & 1.9 & 1.8 & 8.5 \\
\hline mod5adder_128 & 6 & 15 & 4 & 2 & 2.5 & 1.9 & 13.0 \\
\hline decod24-bdd_294 & 6 & 11 & 4 & 2 & 2.6 & 1.8 & 10.2 \\
\hline graycode6_47 & 6 & 5 & 4 & 2 & 1.9 & 1.7 & 5.6 \\
\hline mod5adder_127 & 6 & 21 & 4 & 2 & 2.6 & 1.9 & 16.6 \\
\hline graycode6_48 & 6 & 5 & 4 & 2 & 1.9 & 1.7 & 5.6 \\
\hline mod5adder_129 & 6 & 17 & 4 & 1 & 2.4 & 1.9 & 14.2 \\
\hline decod24-enable_126 & 6 & 14 & 4 & 1 & 2.4 & 1.9 & 12.3 \\
\hline ex3_229 & 6 & 7 & 4 & 1 & 2.4 & 1.8 & 7.2 \\
\hline ex3_228 & 6 & 13 & 3 & 1 & 2.3 & 1.9 & 11.6 \\
\hline ex2_227 & 6 & 13 & 3 & 1 & 2.2 & 1.9 & 11.6 \\
\hline xor5_254 & 6 & 7 & 3 & 2 & 1.9 & 1.8 & 7.2 \\
\hline ex1_226 & 6 & 7 & 3 & 1 & 2.2 & 1.8 & 7.2 \\
\hline decod24-enable_125 & 6 & 9 & 3 & 1 & 1.9 & 1.8 & 8.8 \\
\hline
\end{tabular}

Circuit: Name of the benchmark circuit Standard: Run-time of the standard simulation $n$ : Number of lines Proposed: Run-time of Complexity Impr.: Improvements in the complexity with respect to the worst case $(w c)$ and the best case $(b c)$ compared to the complexity of the standard simulation
[20] M. Saeedi and I. L. Markov, "Synthesis and optimization of reversible circuits - a survey," ACM Computing Surveys, 2011.

[21] R. Drechsler and R. Wille, "Reversible circuits: Recent accomplishments and future challenges for an emerging technology," in Int'l Symp. on VLSI Design and Test, 2012, pp. 383-392.

[22] T. Toffoli, "Reversible computing," in Automata, Languages and Programming, W. de Bakker and J. van Leeuwen, Eds. Springer, 1980, p. 632, technical Memo MIT/LCS/TM-151, MIT Lab. for Comput. Sci.

[23] E. F. Fredkin and T. Toffoli, "Conservative logic," International Journal of Theoretical Physics, vol. 21, no. 3/4, pp. 219-253, 1982.
[24] M. Soeken, S. Frehse, R. Wille, and R. Drechsler, "RevKit: An Open Source Toolkit for the Design of Reversible Circuits," in Reversible Computation 2011, ser. Lecture Notes in Computer Science, vol. 7165, 2012, pp. 64-76, RevKit is available at www.revkit.org.

[25] R. Wille, D. Große, L. Teuber, G. W. Dueck, and R. Drechsler, "RevLib: an online resource for reversible functions and reversible circuits," in Int'l Symp. on Multi-Valued Logic, 2008, pp. 220-225, RevLib is available at http://www.revlib.org. 